\title{
Hop-based Activation Scheduling in Wireless Sensor Networks
}

\author{
Hyunsook Kim ${ }^{1}$ and EunHwa Kim ${ }^{2 *}$ \\ ${ }^{1}$ Faculty of Liberal Education, Daegu University, Gyeongsan, Rep. of Korea, \\ ${ }^{2}$ Graduate School of Education, Yongin University, Korea
}

hs.kim@daegu.ac.kr,ehkimanna@yongin.ac.kr

\begin{abstract}
In a wireless sensor networks, many topics have been studied in several filed. Most of them are concentrated on efficient use of energy to prolong the network lifetime. In a special environment like as remote battlefield, desert, forest, in is more difficult to recharging the battery of sensor. In this paper, we propose a density oontrol method in a dense wireless sensor. We prolong the lifetime of network by selecting small set of active sensor nodes. Hopbased disjoint wakeup method is used and calculates the hop count with adjustment of communication radius. We analyzed the number of active Sensor node and compared with other method with simulation.
\end{abstract}

Keywords: scheduling, energy efficient, wireless sensor network

\section{Introduction}

Wireless sensor network is essential factor of ubiquitous environment. The development of smart sensor technology and wireless communication technology led to many advanced applications such as earth-quake, forest fire, battlefield surveillance, machine failure diagnosis, bioløgical detection, home security, smart spaces and inventory tracking [1-7].

In sensor networks, many topics have been studied in several fields such as MAC, network configuration, query dissemipating, data routing and aggregation, topology, and QoS(Quality of Service). Most of them are seriously investigated for the efficient use of energy in the sensor network to prolong the network lifetime. Sensor node uses battery power for the energy source which is not sufficient for a long time without replacement. In some application scenarios, recharging a battery might be impossible due to the characteristics of sensor network. It can only be equipped with a limited power source. Deploying many sensor nodes is hard to predetermine or engineer because they are sometimes randomly deployed by airplane in inaccessible terrain or in disaster relief operations or in remote battlefield or desert. However, most applications are needed to have a long system lifetime. Therefore, efficient energy use is an important issue in a large-scale wireless sensor networks.

In a multi-hop ad hoc sensor network, each node plays as data originator and data router. The failure of a few nodes can cause significant topological changes and might require packet re-routing and network re-organization. Hence, power management becomes more important and many researchers are currently focusing on the design of energy efficient protocols and algorithms for sensor networks [8-16].

In case of wireless mobile networks, power consumption is not the primary consideration because power resources can be recharged by the user. This network request better quality of

\footnotetext{
${ }^{*}$ Corresponding Author
} 
service (QoS) than the power efficiency. However power efficiency is an important performance metric in sensor networks that directly influences the network lifetime. Application specific protocols can be designed appropriately by trading off other performance metrics such as delay and throughput with power efficiency.

We are focusing on the efficient energy use in the aspect of the network topology configuration in this paper. Some emerging sensor network application scenarios, including battlefield surveillance and agriculture monitoring, involve a large sensor population to monitor a vast, sometimes hostile environment [12]. In a large-scale sensor network, we need to deploy many sensor nodes close together in the sensing field and maintain them in proper way. One of the most important issues in such dense sensor networks is density control [12]. Density control means that it controls the distribution density of the working sensors at certain level [17]. Density control scheme selects working nodes to satisfy the coverage as well as the connectivity. It can save energy by turning off redundant nodes and ean prolong the system lifetime by replacing the failed nodes with some sleeping nodes.

Density control scheme uses a subset of sensor nodes in the active mode. This subset of sensor nodes guarantee two requirements, coverage and connectivity: the area that can be monitored should not be smaller than that of full set of sensor nodes' coverage and the sensor network remains connected so that the data collected by sensor nodes can be delivered to the sink node in order to save power consumption and prolong network lifetime [12]. A practical density control algorithm should be distributed and completely localized because of the distributed nature of sensor networks [18t

We proposed hop-based disjoint wakeup scheduling with selecting additional upstream node [19]. In that paper, we introduced related Works such as GAF, OGDC, ASCENT and Joint Scheduling [17, 20-22]. In this paper, we additionally analyze the proposed method and propose another method.

The rest of this paper iscorgahized as follows. In Section 2, we introduce and analyze three method - the disjointschedyling, two types of the hop-based disjoint wakeup scheme. And we evaluate the proposed algorithm through simulation in Section 3, and finally conclude this paper in Section 4

\section{Scheduling approach}

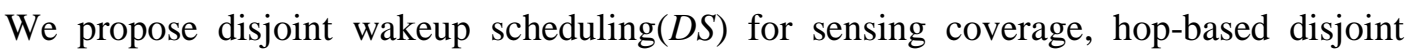
wakeup scheduling with selection of upstream neighbor(HDS-SU)[19], and hop-based disjoint wakeup scheduling with adjustment of hop-radius $(H D S-A H)$.

\subsection{Disjoint Wakeup Scheduling (DS)}

Bisjoint wakeup scheduling is that sensor nodes are waking up if there is no active neighbor within its transmission radius. To avoid the wakeup of sensor node at the same time, each sensor node performs a random back-off by choosing a back-off counter no greater than an interval [23].

Every active sensor node periodically broadcasts $H E L L O \_M S G$ that contains the sensor node identification. Each sensor node receiving HELLO_MSGs constructs information gathered from local broadcast messages, which are the list of active and non-active neighbors. A sensor node determines its activation based on this local information.

Active sensor nodes stay awake continuously and relay data packet within wireless sensor network, while other sensor nodes remain in power-saving mode and periodically check if they should awake and become active sensor nodes. 
In proposed scheme, each active sensor node periodically broadcasts $H E L L O \_M S G$. Sleep sensor nodes also periodically turn on their radios and listen. If a sensor node has been asleep for a while, packets destined for it are buffered at a neighbor. When the sensor node awakes, it can retrieve its packets from the buffering node, typically an active node. A sensor node changes its state between active and sleep.

Periodically, a sleep sensor node switches its state to LISTEN and turn on its radio and checks if it should be active or not. A sensor node switches its state to ACTIVE if it satisfies the active_qualification with local information. Because announcement contention occurs concurrently by several sensor nodes, we resolve this contention with a randomized back-off delay. During random back-off period, each sensor node waits. At the end of this backeoff period, it checks the active_qualification again, and it changes its state to be active if and only if the condition is satisfied. We apply the modified back-off delay as follows [24].

$$
\text { delay }=\left(\left(1-\frac{E_{r}}{E_{m}}\right)+v\right) \times N_{i} \times \mathbb{T}
$$

To rotate active sensor node among all sensor nodes fairly, it continues to be active for a scheduled time period $t_{A}$. The amount of $t_{A}$ is calculated by remained energy $E_{r}$. During its scheduled time, each active sensor node periodically checks if it could change its state to be sleep. A sensor node can go to be sleep mode if it satisfies the sleep_qualification or its $E_{r}$ reaches some proportion of energy.

It informs its withdrawal to its neighbors by stop sending HELLO_MSG for the time period of $t_{L}$. We use the time period of $t_{L}$ as naximum value ol equation (1).

This withdrawal neighbor is treated as a sleep sensor node during this time period. All sensor nodes initially set its state to LISTEN

To analyze the number of active sensor modes in disjoint wakeup scheduling, Figure 1 shows the several cases of two active adjacent nodes in disjoint wakeup scheduling. The distance of two active adjacent nodes ranges from $R+\varepsilon$ to $2 R$.

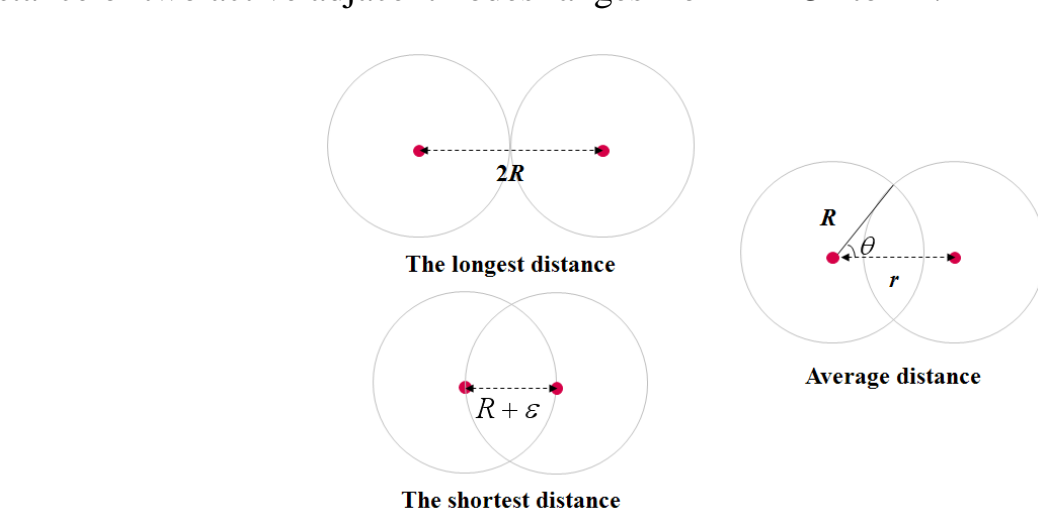

Figure 1. Two cases of adjacent nodes by disjoint wakeup scheduling

Because sensor nodes are uniformly distributed within the network, its distribution over the region can be described by the probability density function (PDF), $f_{\text {node }}(\mathrm{x}, \mathrm{y})$, as follows [25].

$$
f_{\text {node }}(x, y)=f_{\text {node }}(\rho, \theta)=\frac{1}{A}
$$

The cumulative distribution function $(\mathrm{CDF}), F_{\text {dist }}(r)$, of the probability that another node within twice of its coverage has distance $r$ to this node is calculated as follows. 


$$
F_{d i s t}(r)=\frac{\int_{\rho<r} f_{\text {node }}(\rho, \theta) d s}{\int_{\rho<2 R} f_{\text {node }}(\rho, \theta) d s}=\frac{r^{2}}{4 R^{2}}
$$

And the following is PDF.

$$
f_{\text {dist }}(r)=\frac{d F_{\text {dist }}(r)}{d r}=\frac{r}{2 R^{2}} \quad \text { where } 0<r \leq 2 R
$$

The average distance between two active adjacent nodes, $\bar{r}$, can be derived as frollows.

$$
\bar{r}=\int_{R+\varepsilon}^{2 R} r \cdot f_{\text {dist }}(r) d r=\frac{7}{6} R
$$

Using this average distance of two active adjacent nodes, the average number of active adjacent node, $N_{a d j}$, can be calculated as follows.

$$
N_{a d j}=\frac{\pi}{\theta} \approx 3.3 \text { where, } \cos \theta=\frac{72}{R}=\frac{7}{12}
$$

The average number of actixe adjacent node in disjoint wakeup scheduling is 3.3 approximately. To simplify it, we assume that active adjacent nodes are deployed with regular shape. If the value $N_{a d j}$ is six, active sensor nodes are deployed with regular hexagonal shape. Area of regular n-polygon, $A(n)$, is obtained as follows.

$$
A(n)=\frac{a^{2}}{4 * \tan \frac{\theta}{2}} \text { where } \theta=\frac{2 \pi}{n}, \quad a^{2}=2 R^{2}(1-\cos \theta)
$$

The average pure area of a sensor node in a disjoint wakeup scheduling, $A_{\text {disjoint }}$, can be calculated as follows.

$$
A_{\text {disjoint }}=A\left(N_{a d j}\right)=A(3.3)
$$

Using this alue, we derive the average number of active sensor nodes on whole network,

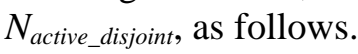

$$
N_{\text {active_disjoint }}=\frac{A}{A_{\text {disjoint }}}
$$

In a dense network, the average number of active sensor nodes in a disjoint wakeup scheduling is not affected by the number of total deployed nodes. The smallest average number of active nodes can be calculated when $n \rightarrow \infty$, in other words $A\left(N_{\text {adj }}\right) \rightarrow 2 \pi R$.

We carry out simulations to measure the number of active sensor nodes in $200 * 200$ square regions with transmission radius as 10 . The average number of active sensor nodes in a disjoint wakeup scheduling converges to value, $N_{\text {active_disjoint }}=255$.

Disjoint wakeup scheduling provides a small number of active nodes and sensor networks and satisfies a high coverage ratio in densely deployed networks. In other words, it provides complete transmission coverage. However, disjoint wakeup scheduling does not guarantee the 
connectivity to the sink node due to the lack of active neighbor nodes with same transmission radius.

The minimum hop counts value will be the length of the shortest path to the sink node [22]. Then the best way to deliver data to the sink node is to send its data to one of its upstream neighbor nodes. To transmit data toward the sink node, a sensor node connects to one of its upstream neighbors. Then we enhance disjoint wakeup scheduling with a hop count.

\section{2. $H D S-S U$}

To investigate the connectivity of an active sensor node with selection of upstream neighbor, we analyze the existence of active upstream neighbor. To analyze the connectivity of active upstream neighbor, a sensor node is simplified as shown in Figure 2.

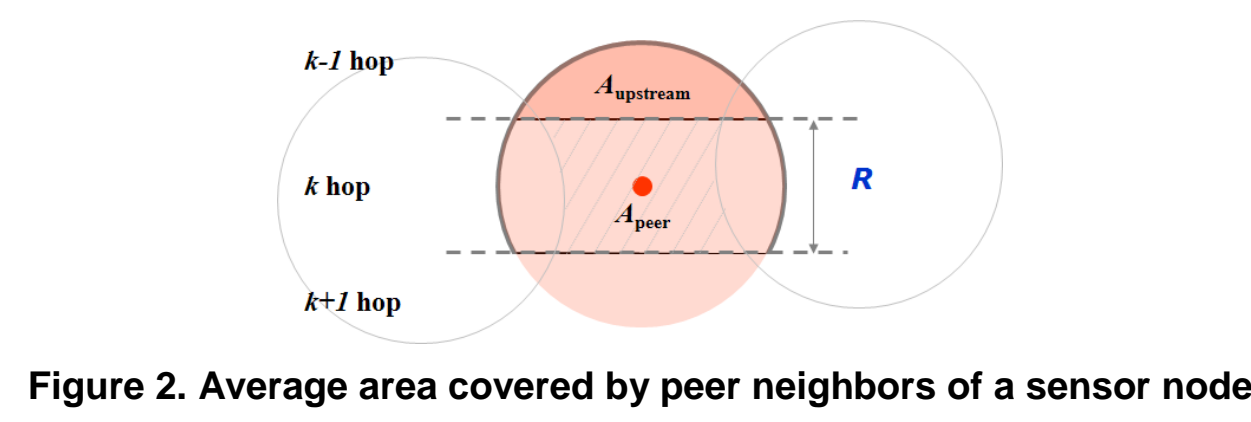

An active sensor node awaked by hop-based disjoint wakeup scheduling is placed over the band of $\mathrm{k}$ hop. To simplify analysis, we consider the center of the height as average point of a sensor node as shown in Figure 2. With this point, we define the count of active upstream neighbor, $C_{\text {active_upstream }}$ as, follows.

The density of upstream neighbor area, $\rho_{\text {upstream }}$, the wakeup probability of a sensor node, $P_{\text {wakeup }}$ can be calculated as below.

$$
\begin{gathered}
\rho_{\text {upstream }}=\frac{A_{\text {upstream }}}{A} * N \\
P_{\text {wakeup }}=\frac{1}{\rho_{\text {peer }}}
\end{gathered}
$$

The density of peer neighbor with hop-based disjoint wakeup scheduling, $\rho_{\text {peer }}$, the area of upstream neighbor, $A_{\text {upstream }}$, and the area of peer neighbor, $A_{\text {peer }}$ can be calculated as below.

$$
\begin{gathered}
\rho_{\text {peer }}=\frac{A_{\text {peer }}}{A} * N \\
A_{\text {upstream }}=2 \int_{\frac{1}{2} R}^{R}\left(\sqrt{R^{2}-y^{2}}\right) d y
\end{gathered}
$$




$$
A_{\text {peer }}=2 \int_{-\frac{1}{2} R}^{\frac{1}{2} R}\left(\sqrt{R^{2}-y^{2}}\right) d y * \frac{A_{\text {disjoint }}}{\pi R^{2}}
$$

From this analysis, we have $C_{\text {active_upstream }}=0.64$ approximately. This value indicates that there is a number of 0.36 that an active node can not find another active upstream neighbor. Actually, the number of upstream neighbors additionally required to become active is not too much burden to the network.

\section{3. $H D S-A H$}

A selection of upstream neighbor provides connectivity to the sink node, but it has two steps for an active node. To provide a more simplified method, we suggest an adjustment of hop-radius. To raise the probability of the existence of active upstream neighbor, we use smaller hop-radius value instead of transmission radius vâlue. In other words, we use decreased transmission radius value just for the calculation of minimun hop count. Also, transmission radius value is used for the other purpose.

We use the value $k$ as a ratio of transmission radus, $R$, and it can vary from 0 to 1 . We use the term, adjusted hop-radius, as the value of $k * R$. If the value $k$ gets smaller value, the probability of the existence of the active upstream neighbor is going to get higher value. However a smaller hop radius also increases the number of disjointed active nodes due to the increased number of hops, but it does notreduce the number of active nodes within each hop. Figure 3 shows a hop-based disjoint wakeup scheduhing with the adjustment of hop-radius as $k=0.5$.

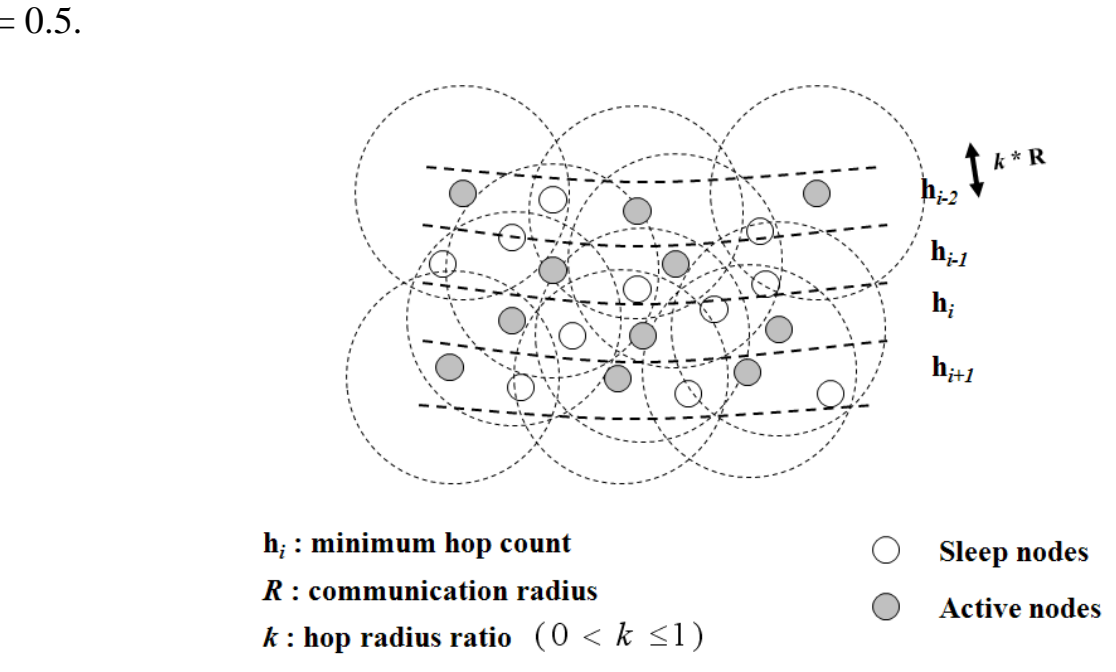

Figure 3. Activation of sensor nodes $(k=0.5)$

In this method, we use the same HELLO_MSG and the state transition diagram of hopbased disjoint wakeup scheduling with the selection of upstream neighbor. In addition, however, every node knows neighbor list using adjustment of hop radius and checks qualification with this neighbor list. 


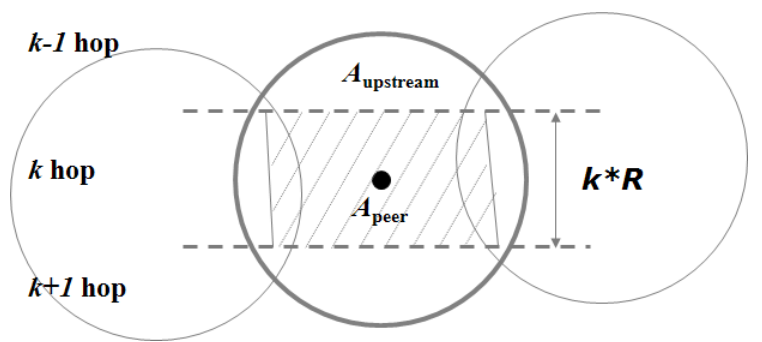

Figure 4. Average area covered by peer neighbors of a sensor node

This method needs a proper value $k$ that meets the existence of active upstream neighbor and minimal number of active sensor nodes. To analyze the connectivity, we caldulate that an active sensor node has how many active upstream neighbors. The connectivity to an active upstream neighbor can be simplified as shown in Figure 4, This figure is the same with Figure 2 except adjusted hop radius, $k * R$.

The connectivity using adjustment of hop radius, Cactive-upstream, is derived from the same formula as described in selection of upstream neighbor.

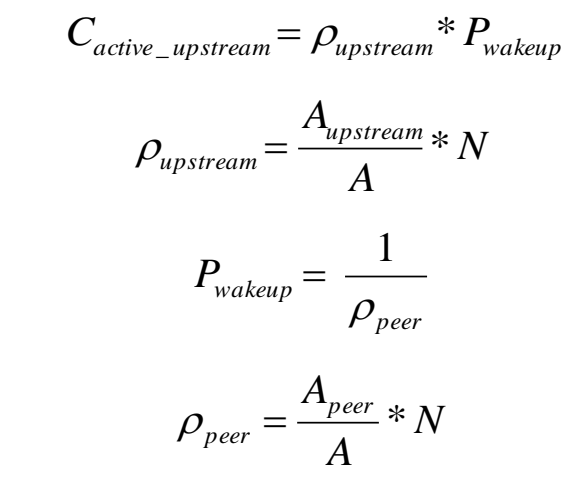

The area of peer neighbor, $A_{\text {peer }}$, and the area of upstream neighbor, $A_{\text {upstream }}$, are calculated as below:

$$
\begin{gathered}
A_{\text {peer }}=2 \int_{-\frac{1}{2} k R}^{\frac{1}{2} k R}\left(\sqrt{R^{2}-y^{2}}\right) d y * \frac{A_{\text {disjoint }}}{\pi R^{2}} \\
A_{\text {upstream }}=2 \int_{\frac{1}{2} k R}^{R}\left(\sqrt{R^{2}-y^{2}}\right) d y
\end{gathered}
$$

The average number of active sensor nodes in a hop-based disjoint wakeup scheduling with adjustment of hop radius, $N_{\text {active }}$, can be calculated as follows.

$$
N_{\text {active }} \approx \frac{A}{A_{\text {peer }}}
$$

Figure 5 shows the average number of active sensor nodes and the connectivity of active upstream neighbors with hop radius $k$. As shown in Figure 5, $C_{\text {active_upstream }}$ and $N_{\text {active }}$ increase exponentially by decreasing of $\mathrm{k}$. To consider the value of $C_{\text {active_upstream }}$ and $N_{\text {active }}$ together, it is sufficient that $C_{\text {active_upstream }}$ is equal to 1 for connectivity. We suggest that hop-based disjoint wakeup scheduling is performed using adjustment of hop radius 0.8 below and perform selection of upstream neighbor for extra wakeup 


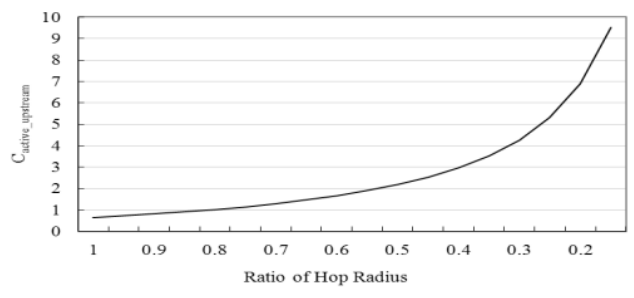

(a) Ratio of connectivity

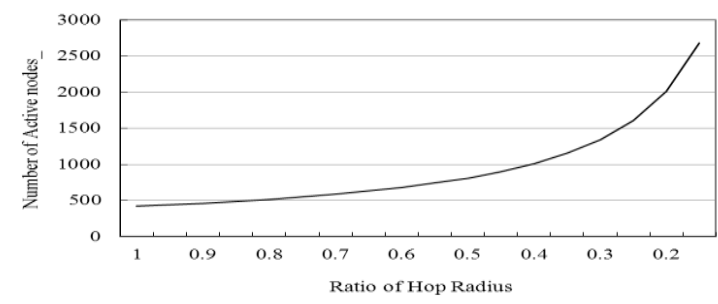

(b) Number of active sensor nodes

Figure 5. Connectivity and number of active sensor nodes with different hop radius

\section{Performance Evaluation}

In this section, we report results from experiments to measure the performance of HDS$A H$. Experiments are carried out on a simulator implemented in C/sova on the top of the 802.11 MAC ad hoc power-saving mode in SPAN [24].

\subsection{Simulation Parameters}

To evaluate the proposed scheme, we deploy sensot nodes over a square region in a uniformly random fashion. The sink node is located at the corner of the sensing field. The parameter sets used for simulation are listed in Table 2. The values listed in Table 2 are default values, so we use gther values of parameters occasionally and in that case, we represent the values separately.

\section{Table 1. Simulation parameters}

\begin{tabular}{|c|c|c|}
\hline A Parameter & Notation & Value \\
\hline Netyyork size & $A$ & $200 * 200$ \\
\hline Number of sensor nodes & $N$ & $200 \sim 2500$ \\
\hline Transmission radio radius & $R$ & 10 \\
\hline Ratio of hop-radius & $k$ & $0.1 \sim 1.0$ \\
\hline
\end{tabular}

\subsection{Simalation results}

In adjustment of hop radius method, we carry out experiments with the hop ratio value $k$ to investigate the influence of the network connectivity. Figure 6 shows transmission coverage, the connectivity of $H D S$ and the connectivity of $H D S-S U$ as the ratio of hop radius decreases.

As shown in Figure 7, the ratio of network connectivity increases as the hop ratio decreases. The smaller hop ratio gives the high connectivity to the upstream neighbors but it also makes more number of active sensor nodes. 

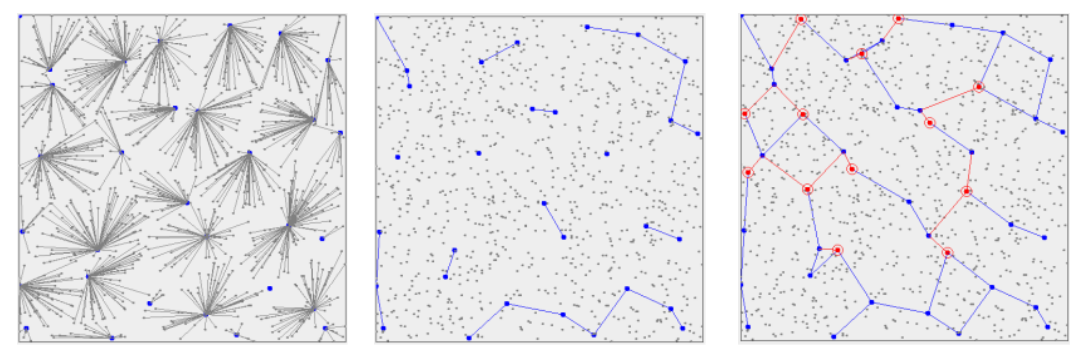

(a) Ratio of hop radius $=1.0$
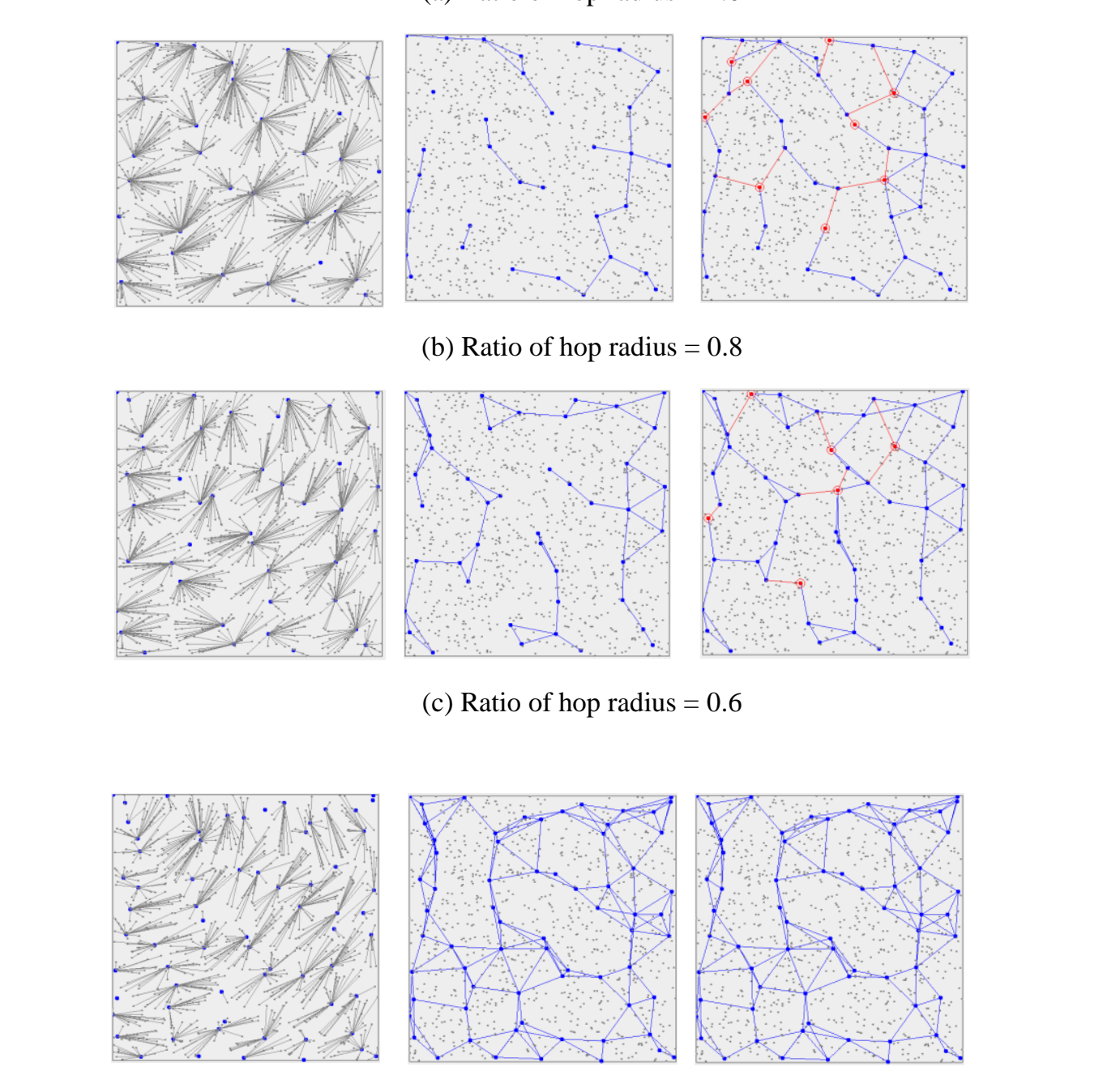

(b) Ratio of hop radius $=0.8$

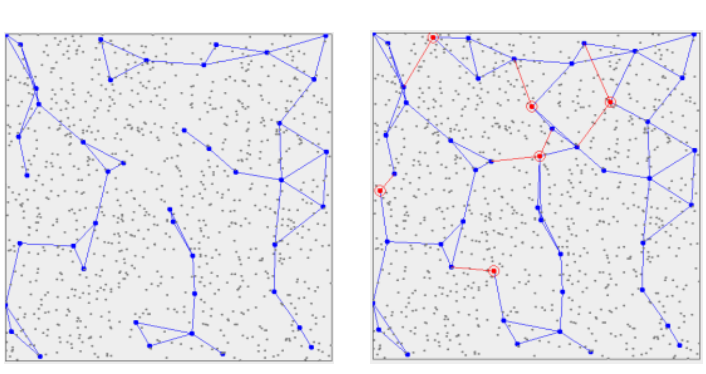

c) Ratio of hop radius $=0.6$
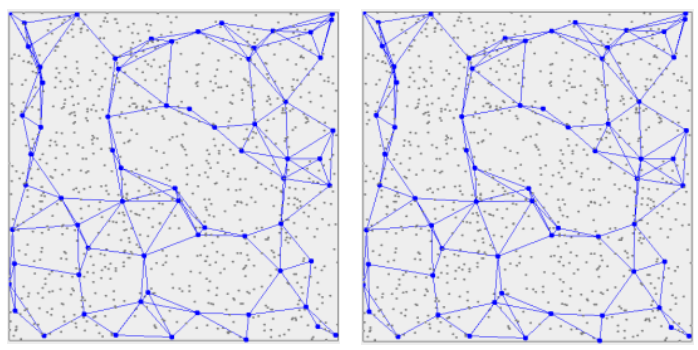

(d) Ratio of hop radius $=0.4$

Figure 6. Network connectivity with different hop radius $(A=500 * 500, R=100, N=1000)$ 

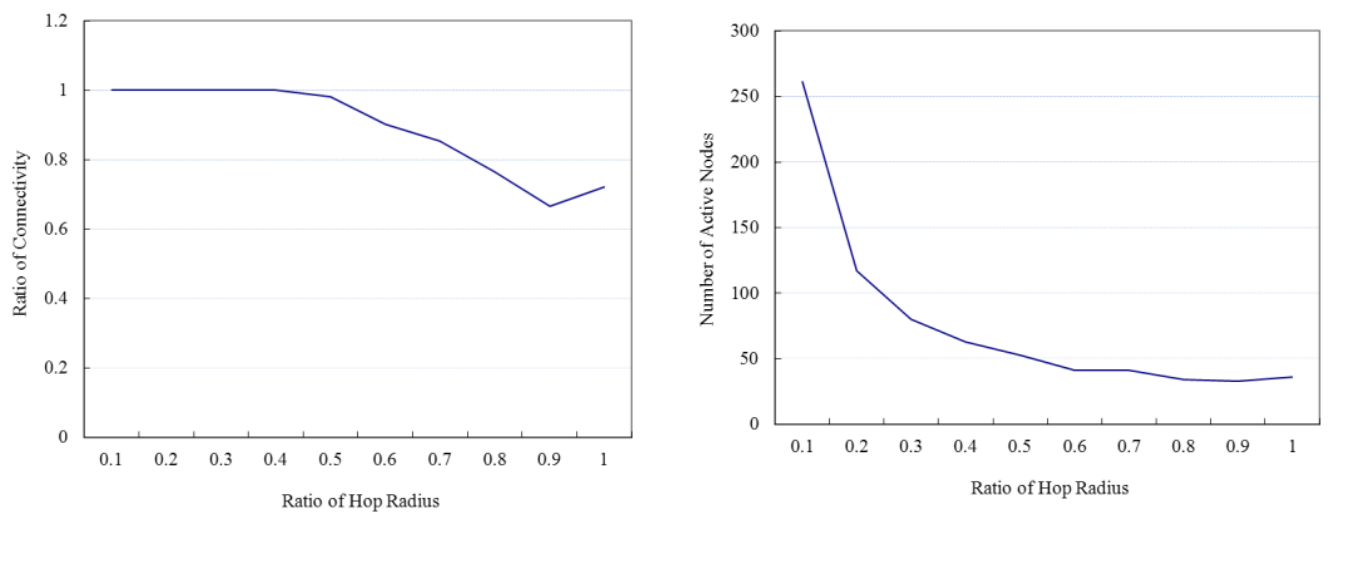

Figure 7. Ratio of network connectivity and density df âctive sensor nodes as ratio of hop radius is varied $(A=500 * 500, R=100, N=3000)$

Figure 8 shows the connected active sensor nodes and the adtive senor nodes with $H D S-S U$ and $H D S-A H$. As hop-radius ratio has smaller value, the number of connected active sensor nodes is growing gradually and the number of active sensor nodes is also growing. Hop-based disjoint wakeup scheduling using adjustment of hop radius provides full connectivity with smallerhop radius ratip and more active nodes than that of the selected upstream neighbor

Figure 8 Density of connected nodes to active sensor nodes as ratio of hop

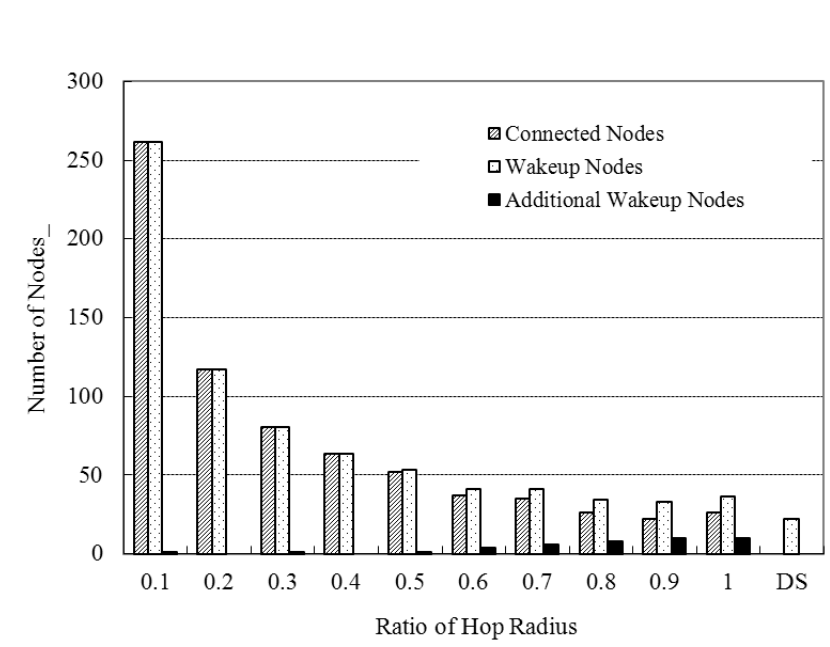
radius is varied $\left(A=500^{\star} 500, R=100, N=15000\right)$

\section{Conclusion}

In this paper, we propose a scheduling method to preserve the energy by selecting a subset of sensor nodes in wireless sensor network field. Disjoint Scheduling method can provide good network coverage with small set of sensor node but can not satisfy network connectivity with same communication radius. Hop-based Disjoint Scheduling method gives good performance with additional method. With adjustment of hop radius, it provides simple way to communication with upstream active sensor node. 


\section{References}

[1] I. F. Akyildiz, W. Su, Y. Sankarasubramaniam, and E. Cayirci, "Wireless Sensor Networks: A Survey", Computer Networks, (2002) March, vol. 38, pp. 393-422.

[2] C. Chong and S. P. Kumar, "Sensor Networks: Evolution, Opportunities, and Challenges", Proceedings of the IEEE, (2003) August, vol. 91, pp. 1247-1256.

[3] H. Kim, "An Efficient Clustering Scheme for Data Aggregation Considering Mobility in Mobile Wireless Sensor Networks", Int'l Journal of Control and Automation, vol. 6, no. 1, (2013), pp. 221-233.

[4] M. Arshad, N. Armi, N. Kamel and N. M. Saad, "Mobile data collector based routing protocol for wireless sensor networks", Scientific Research and Essays, vol. 6, no. 29, (2011), pp. 6162-6175.

[5] C. -M. Liu, C. -H. Lee and L. -C. Wang, "Distributed Clustering algorithms for data gathering in mobile wireless sensor networks", Journal of Parallel and Distributed Computing, vol. 67, (2007), pp. 1187-1200".

[6] A. M. Popescu, I. G. Tudorache, B. Peng and A. H. Kemp, "Surveying Position Based Routing Protocols for Wireless Sensor and Ad-hoc Networks", Journal of Communication Networks and Information Security, vol. 4, no. 1, (2012), pp. 41-67.

[7] N. Ahmad, N. Riaz and M. Hussain, "Ad hoc wireless Sensor Network Architecture for Disaster Survivor Detection”, Int'l Journal of Advanced Science and Technology, v01.34, 2011). pp. 9-16.)

[8] D. Tian and N. D. Georganas, "A coverage-preserving node schedyling scheme for large wireless sensor networks", Proceedings of ACM Wireless Sensor Network and Applícation Workshop, (2002) September, pp. $32-41$.

[9] Md. Zair Hussain, M. P. Singh and R. K. Singh, "Anarysis of Lifetime of Wireless Sensor Network", International Journal of Advanced Science and Technology, (2013) April, vol. 53, pp. 117-126.

[10] R. Sheikhpour, S. Jabbehdari and A. khademzadeh, A A Cluster-Chain-based Routing Protocol for Balancing Energy Consumption in Wireless Sensor Networks", International Journal of Multimedia and Ubiquitous Engineering, (2012) April, vol.7, no.2, pp. 1- 16

[11] C.-S. Nam, K.-S. Jang, G.-S. Choi and D-R.Shin, "Study on Use of a Clustering Technique with ZoneBased Multi-hop Communication in Tireless Sensor Networks", International Journal of Smart Home, (2012) April, vol. 6, no.2, pp. 65-70.

[12] F. Ye, G. Zhong, S. Lu and L. Zhang, 'PEAS: A Robust Energy Conserving Protocol for Long-Lived Sensor Networks", Proceedings of IEEE Network Protocols, (2002) November, pp. 200-201.

[13] H. Huang, G. Hu and F. Yu, "Nergy-aware geggraphic routing in wireless sensor networks with anchor nodes", Int'1. Journal of Communication System's, (2011), pp. 100-113.

[14] E. Park and K. Cho, "Energy Efficient and Reliable Geographic Routing in Wireless Sensor Networks", World Academy of Science, Engineering and Technology, (2010), pp. 631-636.

[15] K. H. Eom, M. C. Kim, S. Y. Park and G. H. Hyum, "The Reduction Method of Power Consumption for the Wireless Sensor Network System", Int'1 Journal of u- and e- Service, Science and Technology(IJUNESST), vol. 6, no. 2, (2013), pp. 6376 .

[16] R. Sheikhpour, S. Jabbehdari and A. Khadem-Zadeh, "Comparison of Energy Efficient Clustering Protocols in Heterogeneous Wireless Sensor Networks”, Int'l Journal of Advanced Science and Technology(IJAST), vol. 36, (2011), pp 27-40.

[17] H. Zhang and J. C. Hou, "Maintaining Sensing Coverage and Connectivity in Large Sensor Networks", Technical Report UIUC, UIUCDCS-R-2003-2351 (2003)

[18] D. Estrin R Govindan, J. S. Heidemann, and S Kumar, "Next century challenges: Scalable coordination in sensor networks," Proceedings of International Conference on Mobile Computing and Networking, (1999) August, pp. 263-270.

[19] E. Kim, "A Density Control Scheme Based on Disjoint Wakeup Scheduling in Wireless Sensor Networks", Int 1 Journal of Smart Home, vol. 7, no. 5, (2013), pp. 327-336.

[20] Y. Xu, S. Bien, Y. Mori, and J. Heidemann, "Topology Control Protocols to Conserve Energy in Wireless Ad Hoc Networks", CENS Technical Report 0006, (2003)

[21] A. Cerpa and D. Estrin, "ASCENT: Adaptive self-configuring sensor networks topologies", IEEE Transactions on Mobile Computing, (2004) July, vol. 3, pp. 272-285.

[22] C. Liu, K. Wu, Y. Xiao, and B. Sun, "Random Coverage with Guaranteed Connectivity: Joint Scheduling for Wireless Sensor Networks", IEEE Transactions on Parallel and Distributed Systems, (2006) June, vol. 17, pp. 562-575.

[23] J. So and N. H. Vaidya, "A Multi-Channel MAC Protocol for Ad Hoc Wireless Networks," UIUC Tech Report, (2003) January.

[24] B. Chen, K. Jamieson, H. Balakrishnan, and R. Morris, "Span: An Energy-Efficient Coordination Algorithm for Topology Maintenance in Ad Hoc Wireless Networks", Proceedings of International Conference on Mobile Computing and Networking, (2001) July, vol. 8, pp. 85-96. 
[25] H. Li and D. Yu, "A statistical study of neighbor node properties in ad hoc network," Proceedings of International Conference on Parallel Processing Workshops, pp. 103-108, Aug. 2002.

\section{Authors}

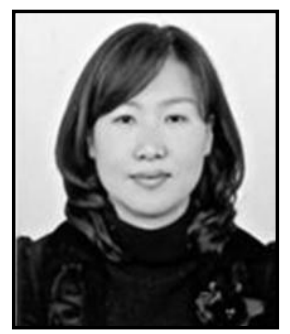

\section{Hyunsook Kim}

She received a B.S. degree in food engineering from Daegu Catholic University, Korea, in 1991 and the M.E. and Ph.D. degrees in computer engineering from Kyungpook National University, Korea, in 2002 and 2007 respectively. Currently, she bas been a professor of liberal education center at the Daegu University, Korea since 2008. Her research interests are various topics of Wireles s Sensor Networks, Wireless Mobile Communications and Vehicle Ad-hoc Networks.

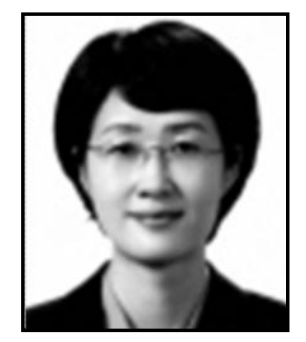

\section{EunHwa Kim}

She received hèr BS degree in Computer Engineering at Kyoungpook National Universify, in 1995. She took her MS in Computer Engineering at Kyoungpook National University in 1999. She receiyed her Ph.D. Computer Engineering at Kyoungpook National Unversity॰in 2008. She was a Software engineer at Samsung Electronics from 1995 to 1996. She is also an Assistant Professor of the graduate school of education at Yongin University from 2012.

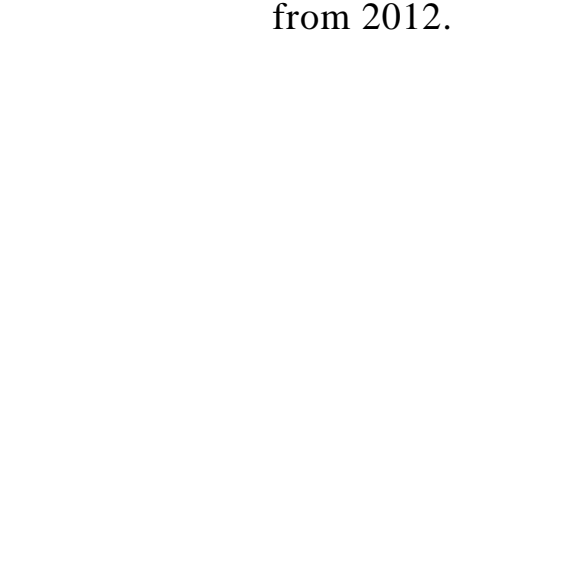

